\title{
Enhancing indoor positioning service for location based internet of things (IoT): a source selecting approach with error compensation
}

\begin{abstract}
This paper presents an enhanced model to handle the radio-frequency (RF) signal to provide a real-time indoor localization service in a practical manner. This model is using the Bluetooth Low Energy (BLE) beacons as the RF signal for indoor localization service. The fingerprinting approach, Received Signal Strength Indicator (RSSI) is used to cut-down the cost of implementation and the accuracy can be further improved with more BLE beacons installed. The RSSI technique applied will be further improved with signal selecting algorithm to determine the right reference points for positioning service. An error compensation algorithm also will be applied to eliminate the false signal which will affect the system's accuracy. This proposed model includes implementation of enhanced RSSI technique with error compensation algorithm to determine the position of users or targeted objects with correct reference points to improve the positioning result.
\end{abstract}

Keyword: Indoor positioning system; Bluetooth low energy (BLE); Fingerprinting; RSSI; IOT devices; Practical approach; Low cost solution; Error compensation; Low power devices; CC2541; CR2032 